\title{
Perspectives and principles of formulation of legal basis of diagnostic quality control of materials and products of nanostructure composites
}

\author{
Gennady K. Baryshev ${ }^{1, *}$, Igor A. Tutnov ${ }^{1,2}$, and Aleksandr P. Biryukov ${ }^{1}$ \\ ${ }^{1}$ National Research Nuclear University MEPhI (Moscow Engineering Physics Institute), Russian Federation \\ ${ }^{2}$ National Research Center "Kurchatov Institute", Moscow, Russian Federation
}

\begin{abstract}
In the paper we discuss the problems of perspective of formulation of legal basis of diagnostics quality control of materials and products of nanostructured composites for various application, including the perspective markets of the National Technology Initiative. We formulate the basic principles including main statements of the typical manufacturing method of diagnostics and quality control of materials and products of nanostructured composites.
\end{abstract}

\section{Introduction}

At present the technological development of Russian Federation is coincided with the realization of the National Technoly Initiative.

The National Technology Initiative (NTI) is a programme aimed at shaping fundamentally new markets and creating conditions for the global technology leadership of Russia by 2035. The NTI includes integrated solutions to define the key technologies, necessary changes in norms and rules, efficient financial and personnel development measures, involvement and encouragement mechanisms for experts with the necessary skills. The choice of technologies is based on major global trends, with network technologies being the priority. Such technologies are people-focused, as people are the end consumers.

The basis for the current systematic work is the NTI matrix which comprises new markets, new technologies, institutes and infrastructure/resources. The NTI areas include a group of the markets of the future:

- EnergyNet (distributed power generation from personal power to smart grid and smart city);

- FoodNet (system of personal production and food and water delivery);

- SafeNet (new personal security systems);

- HealthNet (personal medicine);

- AeroNet (distributed systems of unmanned aerial vehicles);

- MariNet (distributed systems of unmanned maritime transport);

- AutoNet (distributed network of unmanned management of road vehicles);

- FinNet (decentralized financial systems and currencies);
- NeuroNet (distributed artificial elements of consciousness and mentality).

The NTI roadmaps through 2018 shape the ecosystem of future markets. It is important to mention that not all NTI roadmaps have been approved at this moment. As for the implementation of approved roadmaps (NeuroNet and others), this has recently started.

Another important problem being solved is the rapid import substitution of special products of technical purpose. Improvement of systems of life cycle management and planning of new technologies for perspective markets should rely on verified and approved physical basis of products manufacturing, including products for specific application, designed and manufactured on the base of nanostructured composite materials $[1,2]$.

At the moment the so called "smart materials" are of certain interest - these are the materials which operating properties change depending on the operating conditions. For example, this can be a significant increasing (or decreasing) of electrical resistance of the surface by gas sorption from the environment. The operation of semiconductor gas sensors is based on this principle. Another example is the change of transparency of layers by changing of the oxidation state of metals of anode or cathode electro chromic cover, that can be applied for design and development various indicator devices.

Application of "smart materials" - i.e. materials that controllably change their operating properties as a reaction to the change of the environment - in nuclear and other hi-tech industries allows to effectively solve a number of engineering and technical tasks, increase reliability, durability other important technological parameters of products and technical systems. At the moment application of materials with the memory of form effect, sensors of non-destructive testing on the

\footnotetext{
* Corresponding author: GKBaryshev@mephi.ru
} 
base of materials with the application of piezoelectric and magnetostrictive effects is developing [3]. Composite materials for aviation and space industries and markets are no less perspective thematic directions on smart materials.

The range of methods and ways of application of materials and products with the given service properties, including materials bases on nanostructured composites, is respectively wide at the moment [4-6]. At the same time, many of the technologies are still at the stage of research and development, prototypes and industrial samples. The task of formulation of legal technological basis of manufacturing of new products, for the purposes of technological substitution and application at the perspective markets of the National Technological Initiative, including providing the process of quality control with the development of technological maps of such processes for the complete life cycle of items, products and systems, is connected not only with the research of technologies of development of new materials, but also with the development and application of typical manufacturing methods of research and control of service properties of the new class of materials and products during their fabrication.

\section{The general method of research of electrophysical properties of nanostructured composites}

We have developed the general method of research of electrophysical service properties of nanostructured composites.

The development of the method was relied on commonly known and applied methods of measurement of electrophysical properties of conductors [7-9], regarding the following main principles:

1.Principle of verification of the single information field of numerical results of research of electrophysical service properties of nanostructured composites.

This principle provides complete and general enough picture of the set of step processes for real realization of any general method of original methodology of research of electrophysical service properties of nanostructured composite materials or products. This principle in our case defies the mostly close by the criteria of sufficiency of the expected final result physical principles of manufacturing processes in part of application of perspective methods of in-situ research and quality control of composite materials and products. Together with this the principle formalizes the set of information measuring procedures and processes of the applied method of research of electrical resistivity, thermal electromotive force, contact potential difference and other electrophysical properties, defies the sufficient completeness and adequateness of these processes and procedures. The same principle sets the barriers of limitations for research of electric conductivity of nanostructured composites, which should mostly be concentrated on the problems of provision of legal basis of provision of metrological unity of any research process for any quality control of materials and products of that class and provision of the guarantees of high level of accurateness and logical authenticity of the newly obtained information. At the same time, the fundamental principles of the state system of provision of testing measurement unity [10] are applied without change of correcting them.

2. The second principles, in our case of formulation of adequate methods of research of electrophysical service properties of nanostructured composites, is the principle of realization of systematic approach for performing the procedures of virtual measurement and analysis of the information obtained via the procedures. The principle is adopted for the purpose of later imitation modelling of service properties of prototypes and samples of nanostructured composite materials and products and evaluation of the results of such modeling in the form of information data flows on electric resistivity and other parameters, first of all for direct current.

The principle allows to increase the objectiveness of the estimations of the adopted accuracy and authenticity of measured data on phenomenological and numerical levels of information flows of service information of samples of nanostructured composites for research.

3. The third principle of importance for provision of diagnostic measuring procedures for measurement of thermal electromotive force and contact potential difference of nanostructured composite samples is formulated as follows. The principle of priority for object-independent methods of measurement of thermal electromotive force and contact potential difference for nanostructured composite wires and other samples.

The measuring methods for thermal electromotive force and contact potential difference, developed on the given principle, in application to samples of nanostructured composites, would allow to abstract from the featured of quantitate containment and heterogenic allocation of chemical elements through the total of the volume of the samples for measurement.

The application of this principle would allow to take into account only sustainable effects of influence of impurity inclusions on the parameters of thermal electromotive force and contact potential difference of the composite materials and products.

The main step procedures of the developed general method are:

1. Planning the measurement procedure and preparation of the initial data for research of service properties of the samples of nanostructured composites.

2. Development of the documented program of performing of diagnostic research of electrophysical properties of composite samples.

3. Typical instrumental research of electrophysical service properties of nanostructured composites.

4. Estimation of reliability of the results of research of electrophysical service properties of nanostructured composites.

5. Demonstration, validation and presentation of final data results of service properties of the tested samples.

The developed general method takes into account the contemporary image of the features of technological processes of manufacturing of nanostructured composite 
materials and products, degrading of service properties and other perspective items on the duration of their product life cycle, legal requirements for safety, reliability and effectiveness, bases on modernized diagnostic procedures of research of service properties of wires and other composite items, contemporary technical systems and information measuring systems, applies the perfect instruments of commonly used methods of experiment planning, including methods for estimation of errors and reliability at obtaining data of service characteristics of materials and products on the base of nanostructured composites [11].

\section{Development of physical principles of the technology of manufacturing of materials and items of nanostructured composites}

It is important to obtain the methodology and methods of integral engineering and financial analysis of risks of development and application of new measuring and quality control technologies considering the whole process of manufacturing of materials and items of nanostructured composites.

When new measuring systems for quality control of nanostructured composites are designed, it is important to mark the objectives not only for analysis and recognition of engineering problems, but also to prove recommendations to solve this problems, based on rational financial and other costs. The search of optimal solutions at the process of design and implementation of new samples of devices and measuring systems for quality control of materials and products of nanostructured composites, as well as problem definition of new directions of innovation development for economic effectiveness and practical usefulness of diagnostic control of such materials for perspective markets of the National Technology Initiative, require integral and system vision of all possible risks of investment projects [12].

First of all it cares the development of serial production of measuring devices and systems of quality diagnostics for nanostructured composites. For nuclear industry, at the moment, criteria of effectiveness, approved by international practice and recommended by IAEA are applied for estimation of practicability of serial production of measurement techniques for nuclear power plant production [13] - NPV, IRR, DPP - on development of new devices and information measuring systems.

Nevertheless, these economic criteria do not take into account technical risks of investment projects concerning reliability of application of devices and measuring systems in industry. Thereafter the problem of development of scientific categorical apparatus of design of perspective samples of measuring systems with vector-type account integral analysis of engineering and financial risks, mostly for the stages of their development, protoryping and industrial operation. To solve this problem in application to products and materials of nanostructured composites for nuclear industry it is important to specify the basic processes of monitoring of safety of nuclear fuel elements. The next step is to choose the technology of diagnostic control and monitoring of nanostructured materials quality, suggest an innovative and perspective technical device to realize this technology, estimate the practical importance and perspectives of development of a new device or measuring system.

It is also of importance to know possible methods of monitoring and management of engineering and financial risks; to perform the system analysis of main factors of direct and indirect influence on conditions of production of new samples of nanostructured composites.

We in our case understand the following when describing the physical principles of the technology of manufacturing of materials and items of nanostructured composites:

- provision of the main processes of manufacturing preparation in the aspect of development of manufacturing regimes for various items on the basis of research and testing of their electrophysical properties;

- development of technical conditions of provision of inter-operation and final quality control of manufactured products after measurement and testing of their electrophysical properties;

- development of technological maps of technological operations during the process of manufacturing of materials and items of nanostructured composites;

- development of conditions for quality control of the technological process of manufacturing of materials and items of nanostructured composites;

- development of specific instruments, including measuring systems for non-stopping manufacturing quality monitoring of any items of nanostructured composites;

- including in the process the whole cycle of the main process and optional procedures for development and application of technologies of manufacturing of materials and items of nanostructured composites;

- provision the guarantees of safety of the manufacturing technology and final products of nanostructured composites;

- provision of industrial safety and ecological acceptability of manufacturing process of materials and items of nanostructured composites.

Application of the described general method of research of electrophysical properties of nanostructured composites would allow one to provide certification of technical items of materials of such class in industry. Moreover, adopting the service properties of engineering materials of this class to the obligatory safety requirements of contemporary hi-tech industries and perspective markets of the National Technology Initiative require the development of a typical method, including the development of prototypes of information measuring systems of non-destructive testing and technical diagnostics, approbation of the developed general method in the real industrial conditions of environmental influence on materials and items of nanostructured composites. These specific conditions are 
mostly limiting the application of the developed method in the conditions of real manufacturing process in the nearest future.

Still, the developed main principles and steps of the method would allow to make a conclusion about the effective development of principles of the technology of manufacturing of materials and items of nanostructured composites. The described general method may become the basis of the system of quality control management and the system of metrological provision of the future manufacturing and obtaining products with the given quality, as well as the legal basis of diagnostic quality control of materials and products of nanostructure composites

\section{Conclusion}

Formulation of the legal basis of diagnostic quality control of materials and products of nanostructure composites should be based mostly on scientific principles that are the core of the verified and approbated typical industrial methods. We have developed the general method of research of electrophysical properties of nanostructured composites that realizes this principles, has a feature of sufficient scientific novelty, and has all the characteristics of correspondence with the system of provision of unity of measurements and quality control of high technology products.

The results of application of the general method of research of electrophysical properties of nanostructured composites in the context of formulation of physical principles of new technologies of manufacturing of innovation products would allow to gain the new level of development of the system of quality management of such products, provide decreasing the terms of performance in increasing the effectiveness of manufacturing processes of development of such products, as well as increase the controllability and effectiveness of functioning of companies of high-tech industries and perspective markets of the future for all the stages of the life cycle process of materials and products of nanostructured composites.

\section{References}

1. Strategy of the National Security of Russian Federation till 2020, approved by the Decree of the President of Russian Federation of May 12 2009 \#537

2. Decree of the Government of Russian Federation of April 15 2014 \#328 "About the Approving of the State Program of Russian Federation "Development of the Industry and Increasing of Its Competitiveness"

3. V.M. Baranov, A.M. Karasevich. G.A. Sarychev, Testing and quality control of materials and engineering elements (Higher school, Moscow, 2004)
4. V.I. Pantsyrnyy, V.I. Surin, A.P. Biryukov, G.K. Baryshev, E.P. Varyatchenko. Non-ferrous Metals 2 (2014)

5. A. S. Kondrateva, M. Mishin, A. Shakhmin, M. Baryshnikova and S. E. Alexandrov. Phisica Status Solidi 12, 7 (2015)

6. A.I. Voronin, G.K. Baryshev, Yu. V. Bozhko, A.A. Usenko, V. Yu. Zueva, K.I. Litvinova, I.V. Petrova, M.A. Seredina, V.V. Hovailo, Bulletin of the Lebedev Physics Institute 42, 7 (2015)

7. Blatt, Shreder, Foils, Grage, Thermoelectric power of metals (Metallurgia, Moscow, 1980)

8. Anatoly Zharin, Contact Potential Difference Techniques as Probing Tools in Tribology and Surface Mapping (NanoScience and Technology, Springer, New York, 2010)

9. Green, Surface properties of solid state bodies (Mir, Moscow, 1972)

10. RMG 29-99. Russian State System of Provision of Unity of Measurements.

11. Hicks, Basic principles of experiment planning (Moscow, 2013)

12. G.K. Baryshev, I.A. Tutnov. Yu. V. Bozhko, Proceedings of 2016 13th International ScientificTechnical Conference on Actual Problems of Electronic Instrument Engineering (APEIE) (2016)

13. Berens W. Hawranek, Peter M., Manual for the preparation of industrial feasibility studies (UNIDO, Vienna, 1991) 